\title{
Efficient generation of mouse models with the prime editing system
}

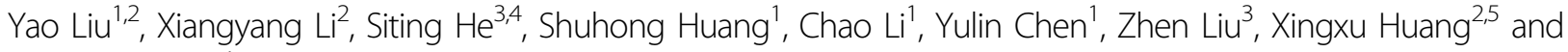 \\ Xiaolong Wang ${ }^{1}$
}

Dear Editor,

Most genetic diseases in humans are caused by singlenucleotide mutations. Although genome editing with either the CRISPR-based cytosine base editor $(\mathrm{CBE})^{1}$ or the adenine base editor $(\mathrm{ABE})^{2}$ holds great promise for gene correction of C-to-T and A-to-G base substitutions in some genetic diseases $^{3,4}$, both editors are useless for correction of other variants such as base transversion, small insertions and deletions (indels).

The prime editing system, a "search-and replace" genome editing technology, was recently added to the genome editing toolkit ${ }^{5}$. The prime editors (PEs) combine an exogenous CRISPR/Cas9 system and endogenous DNA repair system to achieve an increased range of editing versatility, induces all types of base-to-base conversions out of $\mathrm{CBE}$ and $\mathrm{ABE}$ $(\mathrm{C} \rightarrow \mathrm{T}, \mathrm{G} \rightarrow \mathrm{A}, \mathrm{A} \rightarrow \mathrm{G}$, and $\mathrm{T} \rightarrow \mathrm{C})$, small indel, and their combinations. The prime editing system evolved from PE1 to PE3 (PE3b) with stepwise efficiency improvement ${ }^{5}$. The executor of PE1 was constructed by fusing an engineered Cas9 nickase with a reverse transcriptase (M-MLV RTase) ${ }^{5}$, which can target genome sites, nick DNA, and trigger reverse transcription (RT). The executor combining with the engineered prime editing guide RNA (pegRNA) searches for and nicks the target DNA, and thus, new genetic information is encoded into genome by RT. Then, mutations were introduced to M-MLV RTase to improve the editing efficiency of PE1, which is referred to as PE2. Subsequently, in the

\footnotetext{
Correspondence: Zhen Liu (zliu2010@ion.ac.cn) or

Xingxu Huang (huangxx@shanghaitech.edu.cn) or

Xiaolong Wang (xiaolongwang@nwafu.edu.cn)

'Key Laboratory of Animal Genetics, Breeding and Reproduction of Shaanxi

Province, College of Animal Science and Technology, Northwest A\&F

University, Yangling 712100 Shaanxi, China

${ }^{2}$ School of Life Science and Technology, ShanghaiTech University, Shanghai

201210, China

Full list of author information is available at the end of the article

These authors contributed equally: Yao Liu, Xiangyang Li, Siting He
}

PE3 system, to further improve editing efficiency, an additional sgRNA is used to induce nick on the non-edited strand to trigger the endogenous mismatch repair pathway ${ }^{5}$.

In comparison with base editors, PE induces base institutions in more extended regions with fewer bystander mutations ${ }^{5}$. With its unique versatility and accuracy, this technology broadens the scope of genome editing and opens a new avenue for targeted mutagenesis and gene correction in many organisms. However, the efficiency of PE was reported only in five different cell types ${ }^{5}$; it has not been investigated in animals. Here, we demonstrate that $\mathrm{PE}$ can be employed to generate mutant mice with singlenucleotide substitutions.

We first validated the editing versatility of PEs in human HEK293T cells at eight loci (Supplementary Table S1), including two loci (RUNX1 and RNF2) that were reported by Anzalone et $\mathrm{al}^{5}$. PE3 was selected for gene targeting validation, due to its higher editing efficiency compared with PE2 ${ }^{5}$. Sanger sequencing revealed that PE3 induced significant base conversions at six (RUNX1, RNF2, EMX1, $V E G F A, S R D 5 A 3$, and KCNA1) out of eight targeted sites (Supplementary Fig. S1a, b).

PE3 was then used to induce point mutations in the $\mathrm{X}$-linked androgen receptor $(A r)$ gene and the homeobox protein Hox-D13 (Hoxd13) gene in mouse neuro-2a (N2a) cells $^{6,7}$. Both targeted mutations in mice are homologous to human variants associated with clinical diseases in Clin$\mathrm{Var}^{8,9}$. pegRNAs and nick-editing sgRNAs targeting these two genes were designed (Supplementary Table S2). We designed pegRNAs starting with a primer binding site (PBS) length of $13 \mathrm{nt}$ and an RT template length of $\sim 13-15 \mathrm{nt}$. Nicks were positioned $3^{\prime}$ of the edit $\sim 40-60$ bp from the pegRNA-induced nick. Sanger sequencing revealed that PE3 efficiently $(\sim 8-40 \%)$ mediated base transversions at three target sites of Hoxd13 and $A r$ (pegHoxd13-1 for G to C, pegHoxd13-2 for $\mathrm{G}$ to $\mathrm{T}$, pegAr-2 for $\mathrm{G}$ to $\mathrm{T}$ ) (Fig. 1a; 


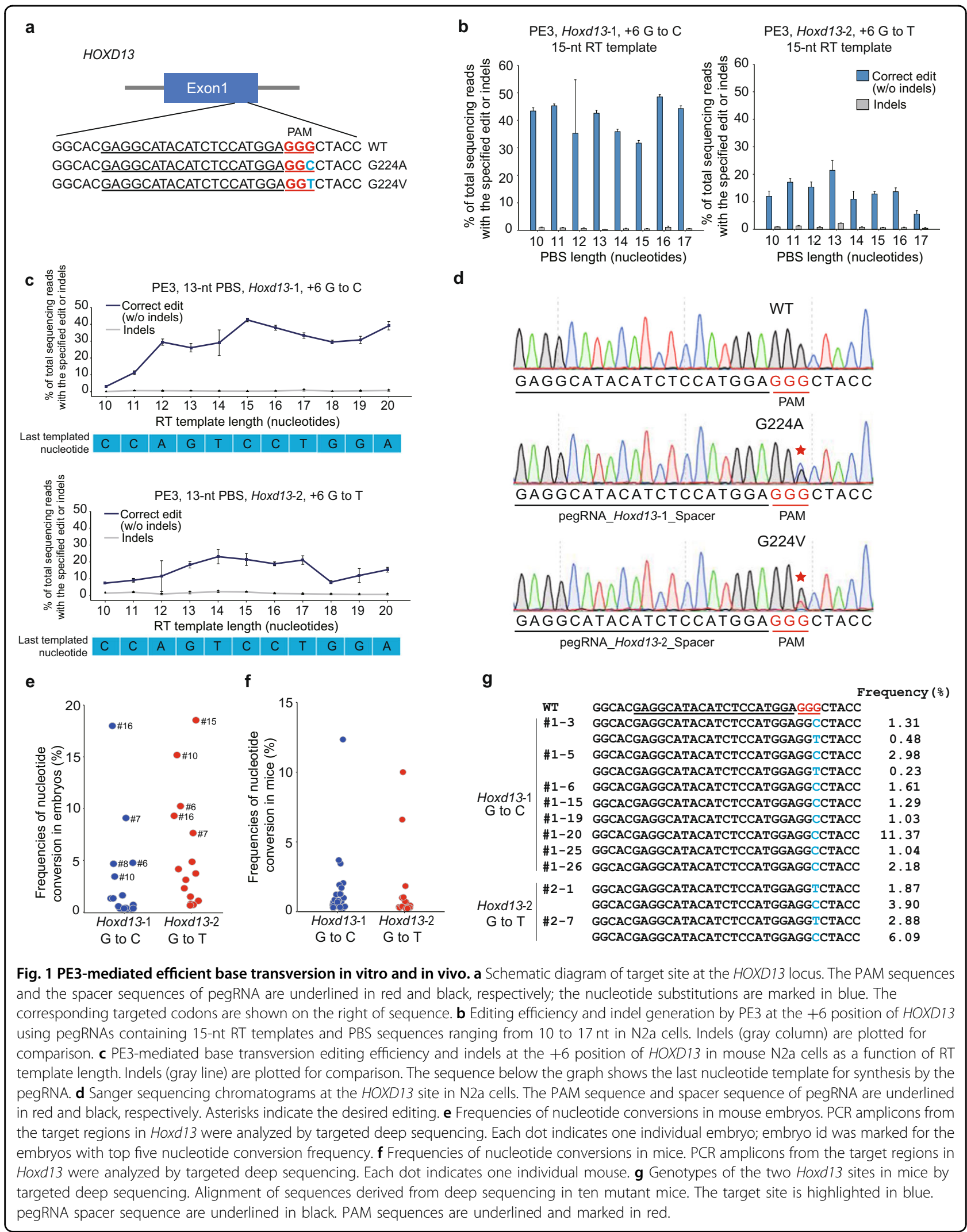


Supplementary Fig. S2a-d). Next, to optimize the performance of pegRNAs, we systematically evaluated pegRNAs with RT template lengths of 10-20 nt and a PBS length of 13 nt for pegHoxd13-1, pegHoxd13-2, and pegAr-2 in N2a cells. Targeted deep sequencing revealed that the editing efficiency of pegRNAs with RT template lengths ranging from 14 to $17 \mathrm{nt}$ were stably efficient in Hoxd13 and $A r$, respectively (Fig. 1c; Supplementary Fig. S3a). Then, using the efficient RT template lengths of 14-17 nt, we systematically evaluated pegRNAs in the context of PBS lengths between 10 and $17 \mathrm{nt}$. Targeted deep sequencing revealed that pegRNAs with a PBS length of 16, 13, and $12 \mathrm{nt}$ for pegHoxd13-1, pegHoxd13-2, and pegAr-2, respectively, had the highest efficiencies; these pegRNAs were used for our in vivo study (Fig. 1b, d; Supplementary Fig. S3b). Anzalone et al. reported that the PE3b system can be applied to the edit lying within a second protospacer to induce fewer indels ${ }^{5}$. Accordingly, we designed nick sgRNA for Hoxd13 based on these parameters and found that the editing efficiency of PE3b was significantly lower than that mediated by PE3 in Hoxd13 (Supplementary Fig. S4), we then selected PE3 for the further analysis.

To edit mouse embryos with PE3, pCMV-PE2encoding mRNA and corresponding nick sgRNA were co-injected with different pegRNAs into one-cell embryos. For Hoxd13, nucleotide conversions were observed in 8 out of 18 (44\%) and 12 out of 16 (75\%) blastocysts, respectively, with mutation frequencies ranging from 1.1 to $18.5 \%$ (Fig. 1e). We observed a low frequency of indels in injected embryos (Supplementary Fig. S5a). Interestingly, we observed not only desirable transversion at the target sites, but also a mixture of nucleotide conversions at these sites (Supplementary Fig. S6), implying a relative low fidelity of the PE3 system in murine embryos that the PE is in need of improved fidelity. Moreover, we observed much lower editing efficiency in embryos for the $A r$ gene (Supplementary Fig. S7), suggesting that screening of pegRNAs in vitro is essential before conducting in vivo studies.

We next delivered mouse embryos that were injected with pCMV-PE2-encoding mRNA and pegRNAs for Hoxd13 into surrogate mothers. Targeted deep sequencing indicated that eight mutant mice out of 30 (Hoxd131 , editing efficiency of $\mathrm{G}$ to $\mathrm{C}>1 \%$ ) and two mutant mice out of 19 (Hoxd13-2, editing efficiency of G to $\mathrm{T}>1 \%$ ) carried mutations at the targeted loci (Fig. 1f, g). Furthermore, the editing efficiency in mutant mice is equivalent to that in prime-edited plants ${ }^{10}$. Together with indel frequency data in embryos and mutant mice (Supplementary Fig. S5), we revealed a low level of indels in the PE system in vivo. As expected, targeted deep sequencing revealed that the editing efficiency varied in the examined nine tissues (heart, liver, spleen, lung, kidney, brain, testis, intestine, and toe) of the two mutant mice (\#1-5 and \#2-14) (Supplementary Fig. S8), indicating that the somatic mosaicism and allelic complexity were induced by zygotic injection. As observed in mouse embryos, we found existence of varied frequencies of undesirable base changes at the two target sites (Fig. 1g).

To determine off-target mutations in PE-edited mice, we identified, using Cas-OFFinder ${ }^{11}$, ten and six putative off-target sites with up to three-nucleotide mismatches and NGG PAM sites for pegRNA and nick sgRNA, respectively. Targeted deep sequencing with genomic DNA from five PE-edited mice $(\# 1-5, \# 1-6, \# 1-20$, $\# 2-14$, and \#2-18) revealed undetectable off-target mutations at all 16 sites (Supplementary Fig. S9a, b).

To comprehensively investigate off-target sites throughout the genome, we conducted whole-genome sequencing for the Hoxd13 mutant mice \#1-26 (G-to-C) and \#2-1 (G-toT). A total of 3,745,168 and 3,840,538 SNPs were identified for \#1-26 and \#2-1, respectively (Supplementary Fig. S10). After filtering out naturally-occurring variants in the dbSNPs database and in two wild-type mice, we picked out the variants with G-to- $\mathrm{H}(\mathrm{H}$ indicates $\mathrm{A} / \mathrm{C} / \mathrm{T})$ and $\mathrm{C}$-to- $\mathrm{H}$ substitutions, and examined whether the remaining SNPs located at the putative off-target sites. Of 3805 predicted offtarget sites, no base substitutions were uniquely found in $\# 1-26$ and \#2-1 (Supplementary Fig. S10). These results demonstrate that the PE3-mediated base conversion is highly specific in vivo.

In sum, we present here the first report of using PEs to generate targeted base conversion mutations in animals. We validate the prime editing system in human cells and demonstrate the versatility of PEs in mice in vivo, though there is a higher frequency of unwanted mutations at target loci. These data support the clinical potential of PEs in correcting a broad range of mutations for genetic diseases.

\section{Acknowledgements}

We are grateful to Dr Perry Hackett for manuscript editing. This work was supported by the National Key R\&D Program (2016YFA0500903 to X.H., 2018 YFC1003000 to Z.L.), the National Natural Science Foundation of China (31972526 and 31772571 to X.W.), as well as Local Grants (2018KJXX-009 to X.W.). X.W. is Tang Scholar at Northwest A\&F University.

\section{Author details \\ ${ }^{1}$ Key Laboratory of Animal Genetics, Breeding and Reproduction of Shaanxi Province, College of Animal Science and Technology, Northwest A\&F University, Yangling 712100 Shaanxi, China. ${ }^{2}$ School of Life Science and Technology, ShanghaiTech University, Shanghai 201210, China. Institute of Neuroscience, CAS Center for Excellence in Brain Science and Intelligence Technology, CAS Key Laboratory of Primate Neurobiology, State Key Laboratory of Neuroscience, Chinese Academy of Sciences, Shanghai 200031, China. ${ }^{4}$ University of Chinese Academy of Sciences, Beijing 100049, China. ${ }^{5}$ CAS Center for Excellence in Molecular Cell Science, Shanghai Institute of Biochemistry and Cell Biology, Chinese Academy of Sciences, Shanghai 200031, China}

\section{Author contributions}

X.H., Z.L., Y.C., and X.W. designed experiments. Y.L., X.L., and S.He performed the experiments. S.Huang and C.L. analyzed the data. All authors discussed the results and approved the manuscript. 


\section{Conflict of interest}

The authors declare that they have no conflict of interest.

\section{Publisher's note}

Springer Nature remains neutral with regard to jurisdictional claims in published maps and institutional affiliations.

Supplementary Information accompanies the paper at (https://doi.org/ 10.1038/s41421-020-0165-z).

Received: 17 February 2020 Accepted: 3 April 2020

Published online: 28 April 2020

\section{References}

1. Komor, A. C., Kim, Y. B., Packer, M. S., Zuris, J. A. \& Liu, D. R. Programmable editing of a target base in genomic DNA without double-stranded DNA cleavage. Nature 533, 420-424 (2016).

2. Gaudelli, N. M. et al. Programmable base editing of $A^{*} T$ to $G^{*} C$ in genomic DNA without DNA cleavage. Nature 551, 464-471 (2017).
3. Zeng, Y. et al. Correction of the Marfan syndrome pathogenic FBN1 mutation by base editing in human cells and heterozygous embryos. Mol. Ther. 26, 2631-2637 (2018).

4. Osborn, M. J. et al. Base editor correction of COL7A1 in recessive dystrophic epidermolysis bullosa patient-derived fibroblasts and IPSCS. J. Investig Dermatol 140, 338-347 (2020).

5. Anzalone, A. V. et al. Search-and-replace genome editing without doublestrand breaks or donor DNA. Nature 576, 149-157 (2019).

6. Radmayr, C. et al. Androgen receptor point mutations as the underlying molecular defect in 2 patients with androgen insensitivity syndrome. J. Urol. 158, 1553-1556 (1997).

7. Dai, L. et al. Mutations in the homeodomain of HOXD13 cause syndactyly type 1-c in two Chinese families. PLoS One 9, e96192 (2014).

8. Landrum, M. J. et al. ClinVar: public archive of interpretations of clinically relevant variants. Nucleic Acids Res 44, D862-D868 (2016).

9. Young, A. I., Benonisdottir, S., Przeworski, M. \& Kong, A. Deconstructing the sources of genotype-phenotype associations in humans. Science $\mathbf{3 6 5}$, 1396-1400 (2019).

10. Lin, Q. et al. Prime genome editing in rice and wheat. Nat. Biotechnol. https:// doi.org/10.1038/s41587-020-0455-x (2020).

11. Bae, S., Park, J. \& Kim, J. S. Cas-OFFinder: a fast and versatile algorithm that searches for potential off-target sites of Cas9 RNA-guided endonucleases. Bioinformatics 30, 1473-1475 (2014). 